\title{
NEW MOLECULAR GENETIC TECHNIQUES: REGULATORY AND SOCIETAL CONSIDERATIONS
}

\author{
K. M. NIELSEN \\ Oslo Metropolitan University, Oslo, Norway; kaare.nielsen@hioa.no
}

\begin{abstract}
SUMMARY
A rapidly expanding toolbox of techniques available for genome editing provides the basis for a new continuum in types of modifications that can be introduced into a genome and blur the bimodal GMO vs. non-GMO (genetically modified organism) divide. Site-directed nucleases (SDN) are now used to modify existing nucleotides within genomes instead of adding recombined DNA as transgenes. Moreover, new gene drive approaches are in development based on SDNs. A number of potential drive applications have been reported, but uncertainties in trait stability and limitations in knowledge of the affected system at various temporal and spatial levels slow down their current uses. Adoption of new genome targeted technology takes place in a social context. The context will vary between countries and cultures, expressed in values, ethics, politics and priorities - that are translated into different regulatory approaches. Some developed products using new genome editing techniques clearly fall under internationally negotiated regulations of GMOs. However, other product outcomes of editing techniques challenge our current understanding and definition of GMOs. There is an urgent need for further research, for building international consensus and harmonizing regulatory approaches to facilitate categorization, predictability, transparency, trust and trade.
\end{abstract}

Key Words: Transgenesis, genetic engineering techniques, genetically modified organisms, GMOs, living modified organisms, site-directed nucleases, CRISPR, gene drive, insect, regulation, genome editing, modern biotechnology, recombinant DNA, stacked transgenes

\section{INTRODUCTION}

\subsection{Current Genetic Techniques}

Established engineering techniques and transgenesis (see Table 1) have provided the basis for the range of genetically modified organisms (GMOs) available for commercial use today. GMOs or living modified organisms (LMOs) are any living organism that possesses a novel combination of genetic material obtained through the use of modern biotechnology (CBD 2000; FAO/IPPC 2017). The majority of these organisms are varieties of commodity crop plants grown in large-scale agriculture. Development and Field Application, pp. 645-654. CRC Press, Boca Raton, Florida, USA. (C) 2021 IAEA 
The globally most commonly used transgenes encode insecticidal properties and herbicide-tolerance. These traits have been obtained through molecular recombination in the laboratory of DNA from several unrelated species. Example of GM-insects reaching field-scale applications are genetically modified male mosquitoes (with a dominant lethal gene) that have been released in various parts of the world (Alphey 2014; Evans et al. 2019) and diamondback moth in limited field releases in the USA (USDA 2017).

The GMO technologies that have reached commercial/field stage applications are based on the introduction of additional DNA to obtain the desired traits. The transgene denotes the inserted recombined DNA, usually consisting of a protein-encoding sequence recombined with several regulatory sequences that ensure expression of a new trait in the engineered organism. Current transgene-enabled technologies and products are typically protected through intellectual property rights (IPR) and sold under contractual agreements. Confidentiality claims limit independent peer review and transparency (Nielsen 2013). Similar constraints can be expected for organisms produced by the new techniques.

Established transgene delivery techniques, such as microinjection, particle gun and Agrobacterium-virulence, result in the random integration of the transgene in the genome of the modified host organisms. Newer commercial versions of plant GMOs are also typically "stacked", i.e. harbour several new-trait encoding transgenes after conventional crossing of plants containing single transgenes/insertion events.

The biological uncertainty caused by the technological opportunity to introduce recombined DNA from several unrelated species into an organism, the unpredictable random insertion of such DNA, the variable levels of containment of the resulting GMO, and the discourse around their cultural and ethical dimensions resulted in early developments of regulatory frameworks on a global scale.

International definitions and regulatory frameworks of GMOs include the Convention on Biological Diversity's Cartagena Protocol on Biosafety (CBD 2000) and the World Health Organization/Food and Agriculture Organization of the United Nations (WHO/FAO) Codex Alimentarius (WHO/FAO 2009). At the national level, GMOs undergo volunteer or mandatory regulation and labelling, depending on the regulatory system in place in the respective country in which they are produced or imported.

\subsection{New Molecular Genetic Techniques}

The rapid development of new genetic techniques provides opportunities for editing existing DNA within genomes at specific locations. This is enabled through for instance the use of site-directed nucleases (SDN). Hence, genetic modifications at particular sites within a genome can be produced without necessarily adding exogenous genes from other organisms or species. The realization of this technologyenabled opportunity is currently limited by biological knowledge of relevant sites and nucleotide alterations that will produce organisms with desired phenotypes.

In addition to the opportunities for limited editing within genomes, SDN can also be used to add recombinant DNA such as transgenes into genomes, but now with sitespecificity. This is in contrast to the random insertions expected from traditional gene- 
delivery techniques. The range of organisms that can be engineered with the new techniques remains somewhat limited to those amenable to traditional genetic modifications due to the need to engineer or produce germline cells with heritable traits.

Besides SDN, other techniques have been developed to engineer genomes at specific sites (Häcker and Schetelig, this volume). When combined with broader conceptual approaches, they may be part of a larger strategy to genetically alter, brand and market a particular organism (Table 1).

Table 1. Various genome-modification techniques, processes and conceptual approaches ${ }^{a}$

\begin{tabular}{|c|c|}
\hline Base editing & $\begin{array}{l}\text { Changing single nucleotides at targeted sites in the genome with site- } \\
\text { directed nucleases (SDN) }\end{array}$ \\
\hline Gene drive & $\begin{array}{l}\text { Mechanism ensuring increase in a defined genotype in a population from } \\
\text { one generation to the next }\end{array}$ \\
\hline Genome editing & $\begin{array}{l}\text { Introducing nucleotide substitutions, insertions, or deletions at targeted sites } \\
\text { with SDN }\end{array}$ \\
\hline Grafting & Process of transplanting rootstock with GM material or vice versa \\
\hline $\begin{array}{l}\text { Intragenesis and } \\
\text { cisgenesis }\end{array}$ & $\begin{array}{l}\text { Production and content of organisms with all-native DNA (intragenesis) and } \\
\text { in addition conserved structure of transferred gene (cisgenesis) }\end{array}$ \\
\hline $\begin{array}{l}\text { Oligo-directed } \\
\text { mutagenesis (ODM) }\end{array}$ & $\begin{array}{l}\text { Transformation of a genome with short oligonucleotides to obtain site- } \\
\text { specific base changes }\end{array}$ \\
\hline Paratransgenesis ${ }^{b}$ & The presence of e.g. GM-microorganisms in the gut of non-GM insects \\
\hline $\begin{array}{l}\text { Recombined or } \\
\text { recombinant DNA }\end{array}$ & $\begin{array}{l}\text { DNA molecules formed by laboratory methods of genetic recombination to } \\
\text { bring together genetic material from multiple sources, creating sequences } \\
\text { that would not otherwise be found in the genome }\end{array}$ \\
\hline Reverse breeding & $\begin{array}{l}\text { Breeding strategy where intermediate organisms are GM but not the final } \\
\text { organism }\end{array}$ \\
\hline RNA interference & $\begin{array}{l}\text { Process where RNA molecules interfere with specific gene expression of } \\
\text { the target organism by neutralizing targeted mRNA molecules }\end{array}$ \\
\hline Synthetic organism ${ }^{c}$ & $\begin{array}{l}\text { Denotes organisms produced through assembled DNA fragments } \\
\text { (synthetically produced) or with extensively modified or transplanted } \\
\text { genomes }\end{array}$ \\
\hline Transgenesis & $\begin{array}{l}\text { Describes the process of inserting recombined and most often unrelated } \\
\text { DNA into a genome, usually at random sites. }\end{array}$ \\
\hline
\end{tabular}

${ }^{a}$ See e.g. Lusser et al. 2011; EFSA 2012a, 2012b; ACRE 2013; AGES 2013; Eckerstorfer et al. 2014

${ }^{b}$ Regulated also as microbial pesticides (see OECD 2004; Aguilera et al. 2011)

${ }^{c}$ Concept relates mostly to microorganisms and industrial production systems

Here I consider two approaches of particular interest for genetic alteration of insect genomes and populations. Firstly, the use of genome editing by SDN for the modification of individual insect genomes, and secondly, the use of gene drive mechanisms to make deliberate changes in the genetic composition of targeted insect populations. 


\section{GENOME EDITING}

\subsection{Site-directed Nucleases}

Enzymes that degrade or generate breaks within a genome are called nucleases. A high number of nucleases are known, of which some have site-specificity. The best known are bacterial restriction enzymes whose discovery initiated the genetic engineering era. Additional types of SDN have more recently been described and further developed for genome engineering purposes; these include the CRISPR-Cas9 system, transcription activator-like effector nucleases (TALEN), zinc finger nucleases (ZFN), and meganucleases. They all offer the opportunity to target genetic alterations to a particular site in the genome. However, they differ in how they recognize the target site, and how and how fast target site-specificity can be changed.

Target site-specificity is due to particular protein motifs (TALEN, ZFN, meganucleases) or RNA sequences (CRISPR-Cas9). The CRISPR-Cas9 system is the most used due to the ease of targeting specific genomic locations by changing the nucleotide composition of the single guide RNA sequence (sgRNA of approx. 20 bases) (Baker 2014; Hsu et al. 2014). Modifications made in the Cas protein has also improved site-specificity, as well as offering the choice between introducing double strand breaks at the target site, versus single-strand breaks and substitutions (Komor et al. 2016; Slaymaker et al. 2016). Due to the broad range of genetic outcomes produced (Hilscher et al. 2017) various names for the technique have been proposed including base, gene or genome editing, and gene targeting.

Nevertheless, since the same SDN technique also can be used to produce larger insertions that clearly fall under current GMO regulations, the use of SDN is not as such indicative of a particular genetic outcome or regulatory pathway. Depending on the extent to which DNA is added in the genome editing process, three classes of outcomes have been described (EFSA 2012b):

- If no new DNA is added in combination with the SDN, spontaneous repair of the double strand breaks in the genome can result in minor nucleotide changes, of which the desired one can be selected for.

- If homologous DNA templates are added with the nucleases, homology-directed repair may result in specific minor nucleotide changes being introduced at the targeted site.

- If larger DNA templates, with or without homologous flanking DNA, are provided with the nucleases, larger DNA fragments encoding several new traits can be inserted.

The nuclease function itself in the engineering process can be delivered as proteins, or encoded by temporal DNA templates, or as part of the inserted DNA.

The DNA repair of the strand breaks produced by the introduced SDN is necessary to obtain the desired genetic alterations. Several natural DNA repair mechanisms are known that deal with spontaneous errors occurring in the genome, such as strand breaks, including non-homologous end joining (NHEJ) of DNA strands and homologous recombination (HR) mediated mechanisms.

The much-desired site-specificity of nucleases is limited by the occurrence of unique target sites (nucleotide compositions) in the genome and the extent the particular nuclease used has off-target activity and unintentionally cleaves at other 
genomic sites with similar nucleotide compositions. The efficiency of DNA repair mechanisms may also vary between species. Whereas nucleases with higher sitespecificity can be engineered, the biology and genetics of the targeted organisms are also important, including knowledge of relevant genetic variation and DNA repair mechanisms. In practical terms, there are also challenges to efficient gene delivery and regeneration systems, as well as chromosome copy number (ploidy), for stable alterations and inheritance.

\subsection{SDN in a Regulatory Context}

Products resulting from genetic techniques, such as SDN, challenge the suitability of the long-held bimodal division of organisms into GMO or non-GMO. A new continuum of product categories now arises because SDN-based engineering is used both in the absence of and in the presence of added DNA sequences. The possible outcomes therefore represent a continuum of engineered organisms with minor changes of existing DNA (e.g. removal of, changes in, or addition of a few nucleotides) to cases where extensive recombined DNA has been added into the genome. Thus, new organisms produced by SDN techniques may span from closely mimicking natural processes occurring through spontaneous mutations to those resembling GMOs produced through recombinant DNA and transgene-based genetic modifications (Podevin et al. 2012). Hence, in some cases the engineered organisms may pose similar biological uncertainties as traditional GMOs when considering the effects of the novel trait(s). In other cases, they are unlikely to be detectable without prior knowledge of the specific nucleotide change introduced. The European Food Safety Authority (EFSA 2012b) has therefore proposed three categories for organism/products produced by SDN techniques (Table 2).

Table 2. Categories for organisms produced by site-directed nucleases (SDN)

\begin{tabular}{|l|l|}
\hline SDN-1: & Contains site-specific random mutations or short deletions \\
\hline SDN-2: & $\begin{array}{l}\text { Contains specific minor nucleotide changes produced by homologous recombination } \\
\text { with an introduced DNA fragment with sequence similarity to the target site }\end{array}$ \\
\hline SDN-3: & $\begin{array}{l}\text { Contains an introduced (often exogenous/recombinant) DNA fragment that was } \\
\text { integrated via non-homologous end joining or homologous recombination }\end{array}$ \\
\hline
\end{tabular}

While SDN-3 clearly falls within the current regulatory framework of GMOs as a recombinant DNA fragment is added to the genome in the process, the regulatory context of SDN-1 and SDN-2 based products remain to be clearly determined and harmonized across countries. Moreover, there is a clear need to develop a uniform taxonomy to ensure uncontested definitions, uniform communication, transparency, and regulatory clarity.

Some major reports on genome editing, SDN and related techniques include European Food Safety Authority (EFSA) guidance (EFSA 2012a, 2012b; Eckerstorfer et al. 2014; Nutfield 2016). 
Site-specific engineering undoubtedly offers increased precision and control of the engineering process. Thus, a reduction in the biological uncertainty arising from the engineering process itself is expected. Some process related uncertainty may nevertheless remain; for instance, from the variable consistency of the outcomes of the suite of related SDN techniques applied across laboratories, and on different genomes, species, regeneration systems, and traits. Thus, some of the uncertainty arising from the engineering process, that formed part of the rationale for the current regulation of GMOs, is not a priori absent from all products produced by SDN.

Issues resembling those considered in the assessments of GMOs that may materialize in some applications of the new techniques are:

1. Incorporation of recombined DNA from multiple/unrelated species

2. Co-integration of vector sequences used for technology delivery

3. Off-target cleavage causing random breaks and genome rearrangements

4. Modified/insertion sites containing new open reading frames

5. Variable expression and stability of intended traits

6. Available history of safe use of traits (e.g. in donor versus new recipient), and

7. Effect of new trait on host biology.

The relevance of the above issues must be assessed on a case-by-case basis. The broad utility of SDN-based engineering approaches has clearly contributed to the broader discourse on process versus product-triggered regulation (Sprink et al. 2016). The process-based approach is triggered in a regulatory context by the techniques used, and the product-based approach is triggered by the novelty of the product.

In conclusion, SDN-based products may or may not resemble traditional GMOs and a globally harmonized approach has not yet been negotiated. A key issue is to reach a shared understanding of a process-based versus a product-based approach to risk and regulation. Risk assessment and regulation of SDN-based products that are not considered covered by current regulation of GMOs is currently done case-by-case at the national level.

\section{GENE DISPERSAL AT THE POPULATION LEVEL}

\subsection{Self-sustaining Mechanisms for Gene Dispersal in Wild Populations}

Gene drive is a mechanism that increases a specific genotype from one generation to the next-by ensuring non-Mendelian inheritance of chromosomes during sexual reproduction. Gene drives are naturally occurring mechanisms that have been adapted for purposes of genetic engineering of wild populations. Noteworthy, the genetic engineering approach is deliberately developed to be self-sustaining, e.g. to ensure the spread of defined genetic material through a natural population in the environmentin sharp contrast to most other self-limiting genetic engineering approaches today that seek to document containment of the engineered trait in cultivated or domesticated species.

New gene drive systems rely on the opportunities presented by SDN (CRISPRCas9) for the intentional spread of genes in wild populations (Esvelt et al. 2014 and references within). Gene drive systems today mostly remain at the idea/ developmental stage in which the potential usages of the technology in the field are 
being explored. A key limitation for the broad uptake of gene drive systems include the extent the traits are sufficiently stable once present in wild populations (Callaway 2017).

It is noted that population control techniques such as the use of sterility (mainly induced by radiation) are not currently relying on gene drives. The Sterile Insect Technique (SIT) acts at the population scale, based on the systematic area-wide release of sterile insects that outcompete (in numbers) the fertile insects of the target pest population. The result being that the offspring is non-viable and the population declines. The SIT has been successfully employed for many decades and relies on radiation-based sterilisation (Dyck et al. 2021).

More recently, inducible male sterility has been achieved by recombinant DNA and traditional genetic engineering that results in mosquitoes and agricultural pests regulated as GMOs (Alphey 2014; WHO 2014; USDA 2017).

\subsection{Gene Drive Systems in a Regulatory Context}

The comparative approach that has proven useful in framing the assessment of selflimiting GMOs may be of little utility to assess intentional spread of self-sustaining DNA in wild populations by gene drive systems. The environmental impact assessment of gene drive technology requires robust knowledge of the affected population's structure, size, behaviour, migration patterns, reproduction and generation time. Moreover, the assessment also rests on a robust knowledge of the population's interactions with other organisms at various trophic levels in the receiving environment (see for instance USDA 2017). Limited availability of data on these aspects often reduces the opportunities for data-driven risk assessments (see van Lenteren et al. 2006).

It is currently not clear who is responsible for generating data that would allow a more in-depth understanding of wild populations targeted for gene drive approaches (e.g. agricultural or human health pests). It is also not fully clear how biological data collected at one location can be considered relevant for other areas. This will have an impact on the validity of risk assessment conclusions, that under situations of limited data, may partly rely on extrapolation between species, populations and environments. Trait stability is also a potential issue and resistance to an introduced gene drive mechanism in populations may develop (Callaway 2017).

Finally, gene drives must be considered in a tempo-spatial context. The spatial component also includes knowledge of a drive's dissemination pattern over time, within and between populations, and the potential for cross-border migration of gene drive-modified organisms.

Broad reviews of concepts and applications prior to release of GMOs with a gene drive system, as well as public discussions of technology that has the potential to affect the global commons, have been called for (Oye et al. 2014). An extensive report by the National Academy of Sciences of the USA (NAS 2016) concluded that there is insufficient evidence available to support the release of gene drive-modified organisms into the environment at this time. However, the report also acknowledges that gene drives have significant potential for basic and applied research, including implementing highly controlled field trials (NAS 2016). 
In conclusion, current gene drive proposals are limited by uncertainties in the knowledge of the affected system and the target populations. Most new proposals rely on introducing self-limiting SDN-based transgenes and will fall under current GMO regulations. Many gene drive-modified organisms are yet at the exploratory stage and initial field releases will be regulated according to national GMO legislation.

\section{TECHNOLOGICAL DEVELOPMENTS IN CONTEXT}

Genome editing by SDN have been called a transformative and disruptive technology. The degree and speed of adoption of new technologies nevertheless takes place in a social context (Ishii and Araki 2017). That social context will vary between countries and include differences in values, ethics, politics and priorities. Differences in these variables translate into differences in regulation. Understanding drivers of technological innovation and broadly accepted frames for benefit-cost analyses is also of importance and may vary with culture and market opportunities.

The social context also includes the role of expert and expert cultures, which defines valid concerns for risk assessment and how scientific uncertainty and knowledge gaps are addressed and communicated at various levels (Nielsen and Myhr 2007).

Furthermore, standards and principles for approaches to risk and uncertainty are negotiated at both the national and international levels. Currently, such approaches are usually limited to defined biological risks, and internationally harmonized approaches to the broader issues encompassed in benefit-cost analyses are not yet available.

The broader issues include the ethical dimensions of importance for a broader societal acceptance. For instance, a clear understanding of how risk and benefits are or can be distributed among various stakeholders is needed (Nutfield 2016; ECNH 2016). In this context, there are both calls for more proportionate treatment of risks in a process versus product-based perspective, as well as calls for the need of balancing the exposure of risks with the beneficiaries.

In all cases, different types of uncertainty and how they are handled represent an integral component of the process of technology introductions. Procedures for transparency in the treatment and communication of risks identified in assessment have recently been proposed (EFSA 2017). Improved approaches to handling and communicating uncertainty may substantially improve transparency of processes and help further public trust in procedures.

The way forward for the technological opportunities presented here will be found in the landscape of biological, regulatory and political uncertainty as shaped by further scientific advances, values, and culture. The level of public trust created through the framing of risk, engagement and transparency seems essential to optimize the nonlinear trajectory between technological opportunity and adoption. 


\section{REFERENCES}

(ACRE) Advisory Committee on the Releases to the Environment. 2013. ACRE advice: New techniques used in plant breeding. UK. $44 \mathrm{pp}$.

Alphey, L. 2014. Genetic control of mosquitoes. Annual Review of Entomology 59: 205-224.

(AGES) Austrian Agency for Health and Food Safety. 2013. New plant breeding techniques. RNAdependent methylation, reverse breeding. Federal Ministry of Health. Vienna, Austria. 72 pp.

Aguilera, J., A. Gomes, and K. M. Nielsen. 2011. Genetically modified microbial endosymbionts as insect pest controllers: Risk assessment through the European legislations. Journal of Applied Entomology 135: 494-502.

Baker, M. 2014. Gene editing at CRISPR speed. Nature Biotechnology 32: 309-312.

Callaway, E. 2017. Gene drives meet the resistance. Evolution could weaken technique's potential in the wild. Nature 542: 15.

(CBD) Convention on Biological Diversity. 2000. Cartagena Protocol on Biosafety to the Convention on Biological Diversity: Text and annexes. Secretariat of the Convention on Biological Diversity. Montreal, Canada. 31 pp.

Dyck, V. A., J. Hendrichs, and A. S. Robinson (eds.). 2021. Sterile Insect Technique - Principles and practice in Area-Wide Integrated Pest Management. Second Edition. CRC Press, Boca Raton, Florida, USA. 1200 pp.

Eckerstorfer, M., M. Miklau, and H. Gaugitsch. 2014. New plant breeding techniques and risks associated with their application. Report 0477, Umweltbundesamt GmbH. Vienna, Austria. 92 pp.

(ECNH) Federal Ethics Committee on Non-Human Biotechnology. 2016. New plant breeding techniques - Ethical considerations. Bern, Switzerland. 32 pp.

(EFSA) European Food Safety Authority. 2012a. Panel on Genetically Modified Organisms (GMO: Scientific opinion addressing the safety assessment of plants developed through cisgenesis and intragenesis. EFSA Journal 10: 2561.

(EFSA) European Food Safety Authority. 2012b. Panel on Genetically Modified Organisms (GMO): Scientific opinion addressing the safety assessment of plants developed using Zinc Finger Nuclease 3 and other Site-Directed Nucleases with similar function. EFSA Journal 10: 2943.

(EFSA) European Food Safety Authority. 2017. Scientific committee guidance on uncertainty in EFSA scientific assessment, scientific opinion. Draft version, European Food Safety Authority, Parma, Italy, $211 \mathrm{pp}$.

Esvelt, K., A. L. Smidler, F. Catteruccia, and G. M. Church. 2014. Emerging technology: Concerning RNA-guided gene drives for the alteration of wild populations. eLife 3: e03401.

Evans, B. R., P. Kotsakiozi, A. L. Costa-da-Silva, R. S. Ioshino, L. Garziera, M. C. Pedrosa, A. Malavasi, J. F. Virginio, M. L. Capurro, and J. R. Powell. 2019. Transgenic Aedes aegypti mosquitoes transfer genes into a natural population. Scientific Reports 9: 13047.

(FAO/IPPC) Food and Agriculture Organization of the United Nations/International Plant Protection Convention. 2017. Glossary of phytosanitary terms. International Standard for Phytosanitary Measures (ISPM) No. 5. IPPC, Rome, Italy.

Hilscher, J., H. Bürstmayer, and E. Stoger. 2017. Targeted modification of plant genomes for precision crop breeding. Biotechnology Journal 12: 1600173.

Hsu, P. D., E. S. Lander, and F. Zhang. 2014. Development and applications of CRISPR-Cas9 for genome engineering. Cell 157: 1262-1278.

Ishii, T., and M. Araki. 2017. A future scenario of the global regulatory landscape regarding genomeedited crops. GM Crops \& Food 8: 44-56.

Komor, A. C., Y. B. Kim, M. S. Packer, J. A. Zuris, and D. R. Liu. 2016. Programmable editing of a target base in genomic DNA without double stranded DNA cleavage. Nature 533: 420-424.

Lusser, M., C. Parisi, D. Plan, and E. Rodriquez-Cerezo. 2011. New plant breeding techniques. Stateof-the-art and prospects for commercial development. Joint Research Center, European Commission EUR 24760 EN. Publications Office of the European Union, Luxembourg.

NAS (National Academy of Sciences). 2016. Gene drives on the horizon: Advancing science, navigating uncertainty, and aligning research with public values. The National Academies Press. Washington, DC, USA. 230 pp.

Nielsen, K. M. 2013. Biosafety data as confidential business information. PLoS Biology 11: e1001499. 
Nielsen, K. M., and A. Myhr. 2007. Understanding the uncertainties arising from technological interventions in complex biological systems: The case of GMOs, pp. 108-122. In T. Traavik, and L. L. Ching (eds.), Biosafety first: Holistic approaches to risk and uncertainty in genetic engineering and Genetically Modified Organisms. Tapir Academic Press, Trondheim, Norway.

(Nutfield) Nutfield Council on Bioethics. 2016. Genome editing: An ethical review. Nuffield Council on Bioethics, London, UK. 136 pp.

(OECD) Organisation for Economic Cooperation and Development. 2004. Guidance for information requirements for regulation of invertebrates as biological control agents. OECD Series on Pesticides 21, JT00156791. OECD, Paris. 22 pp.

Oye, K. A., K. Esvelt, E. Appleton, F. Catteruccia, G. Church, T. Kuiken, S. B-Y. Lightfoot, J. McNamara, A. Smidler, and J. P. Collins. 2014. Regulating gene drives. Science 345: 626-628.

Podevin, N., Y. Devos, H. V. Davies, and K. M. Nielsen. 2012. Transgenic or not? No simple answer! EMBO Reports 13: 1057-1061.

Slaymaker, I. M., L. Gao, B. Zetsche, D. A. Scott, W. X. Yan, and F. Zhang. 2016. Rationally engineered Cas9 nucleases with improved specificity. Science 351: 84-88.

Sprink, T., D. Eriksson, J. Schiemann, and F. Hartung. 2016. Regulatory hurdles for genome editing Process- vs. product-based approaches in different regulatory contexts. Plant Cell Reporter 35: 14931506.

(USDA) United States Department of Agriculture. 2017. Proposal to permit the field release of genetically engineered diamondback moth in New York. Environmental assessment April 2017. USDA-APHIS, Riverdale, Maryland, USA.

van Lenteren, J. C., J. Bale. F. Bigler. H. M. T. Hokkanen, and A. J. M. Loomans. 2006. Assessing risks of releasing exotic biological control agents of arthropod pests. Annual Reviews of Entomology 51: 609-634.

(WHO) World Health Organization. 2014. Guidance framework for testing of genetically modified mosquitoes. WHO, Geneva, Switzerland.

(WHO/FAO) World Health Organization/Food and Agriculture Organization of the United Nations. 2009. Foods derived from modern biotechnology. Second edition. FAO, Codex Alimentarius Commission, Joint FAO/WHO Food Standards Programme. Rome, Italy. 76 pp. 